\title{
Spin Glass Phase Exists in the Random Weak Disorder for the Villain Model
}

\author{
Md. Yeakub Ali*, Sunil Dhar \\ Department of Mathematics, Chittagong University of Engineering and Technology, Chittagong, Bangladesh \\ Email: ${ }^{*}$ ali69cuet@gmail.com, sdhar@cuet.ac.bd
}

Received 5 August 2014; revised 19 September 2014; accepted 7 October 2014

Copyright (C) 2014 by authors and Scientific Research Publishing Inc.

This work is licensed under the Creative Commons Attribution International License (CC BY). http://creativecommons.org/licenses/by/4.0/

(c) (i) Open Access

\begin{abstract}
In this work we have studied non random Villain model by introducing simple defects to calculate degeneracies of the first excited states using Pfaffian approach through a perturbation theory. The distributions of excitations of the ground states are displayed graphically. The results are indicated that spin glass occurs in the weak disorder for the Villain model. At the concentration of defect bonds $p=0.03$, the distribution behaves in the same manner as for $p=0.5$ for different sizes of lattice. The latest result of the spin glass is presented in this paper.
\end{abstract}

\section{Keywords}

Spin Glass, First Excitations, Distribution, Square Lattice

\section{Introduction}

In this paper we have presented computational results for excitations of ground states for the Villain model [1] by introducing simple defects. In the Villain model, we have replaced ferromagnetic bonds by antiferromagnetic bonds randomly and calculated first excitations in the concentrations of defect bonds $p=0.01$ to 0.5 for the whole random system. The initial model is fully frustrated [2] with highly degeneration of ground states and the replacement of ferromagnetic bonds with antiferromagnetic bonds reduces frustration [3]. The spin glasses are disorder system [3] which is defined as the random combinations of ferromagnetic and antiferromagnetic bonds.

We have expected the Villain model to have a spin glass at zero temperature in the aforementioned limit of weak disorder. In this study, the square lattice $L \times L$ is wounded cylindrically and the periodic boundary condition is in one dimension. The energy gap is $4 J$ in the mentioned system if $L$ is even. Otherwise it is $2 J$ [4] [5]. In this work, we propose a simple picture of spin glass phase in the weak disorder that has evaluated from power law distribution of first excitations of ground states.

*Corresponding author. 
An outline of this paper is as follows. In Section 2 we introduce formalism and discuss with model and method how one can calculate first excitations. In Section 3 we present results from numerical simulation of the Villain model with antiferromagnetic exchange randomness and in Section 4 we draw concluding remarks.

\section{Formalism}

The frustration of two dimensional Ising model with interaction in one dimension is studied in this paper extensively. The two dimensional fully frustrated Ising model, or Villain model [1], consists of Ising spins on a square lattice with nearest neighbor bonds $\left|J_{i j}\right|=1$ with periodic boundary conditions. The Hamiltonian can be written as

$$
H=-\sum_{\langle i j\rangle} J_{i j} \sigma_{i} \sigma_{j}
$$

where $\sigma_{i}= \pm 1$ are Ising spins on a square lattice, $L \times L$ as shown in Figure 1 .

The method is used in the calculation basing on the Pfaffian [5] through a degenerate state perturbation theory [6]-[8]. The degeneracies of the excitated states are calculated from an expression of the eigenvalues with disorder subspace. We write the partition function for the two dimensional Ising model as

$$
Z=2^{N}\left[\prod_{\langle i j\rangle} \cosh \left(J_{i j} / k T\right)\right](\operatorname{det} D)^{1 / 2}
$$

where $J_{i j}$ is the nearest-neighbour bond strength for sites $\langle i j\rangle$ and the product is over all bonds on the $N$-site lattice. The Matrix $D$ [9]-[15] is Hermitian, pure imaginary, and of order $4 N$. The eigenvalue of Matrix $D$ can be written

$$
\varepsilon= \pm \frac{1}{2} X \exp (-2 J r / k T)
$$

where $r$ is an integer and $X$ is a real number. At $T=0$ there is a degeneracy at $\varepsilon=0$ equal to the number of frustrated plaquettes. The expression the ground-state degeneracy are written a

$$
M_{0}=\prod_{d} X_{d}
$$

where $M_{i}$ is the degeneracy of the $i$ th excitated states. The entropy of the bimodal spin glass Ising model can be expressed in terms of the degeneracies as

$$
S=\ln \left(2 M_{0}\right)+\frac{M_{1}}{M_{0}} \mathrm{e}^{-4 J / T}+\frac{M_{1}}{M_{0}} \frac{4 J}{T} \mathrm{e}^{-4 J / T}+\cdots
$$

The specific heat of the system can be written as

$$
C=\left(\frac{4 J}{T}\right)^{2} \frac{M_{1}}{M_{0}} \mathrm{e}^{-4 J / T}+\cdots
$$

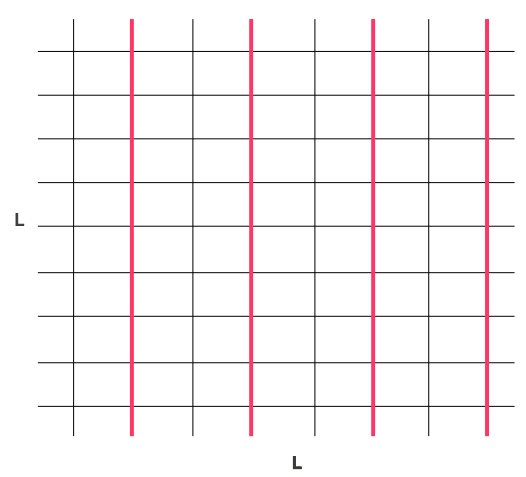

Figure 1. The Villain model on $L \times L$ lattice; along the horizontal direction, bonds are ferromagnetic while along vertical direction, bonds are ferromagnetic and antiferromagnetic alternately. 


\section{Results and Discussion}

We have carried out a numerical calculation to lift degeneracies of excited states at zero temperature. The disorder is confined to a frustrated square patch with periodic boundary conditions in one dimension. Square lattices of size $L \times L$ were considered over a range of concentrations of negative bond $p \in[0.01,0.5]$. As $p$ for the Villain model is zero, the concentration of negative bonds increases from $p=0.01$ to 0.5 . We have shown that there is a mechanism from the Villain phase to spin glass phase, which is characterized by the excitations of ground states. These excitations can exist for the disordered spin glass on the finite system. We have calculated $\frac{M_{1}}{M_{0}}$ for lattice sizes and several concentrations for Villain model. The distribution of excitations for $p=0.01$ are the most likely value in the scale $L^{2} \ln L$ as shown in Figure 2. It has been shown that the distributions of degeneracy of the first excited state are fat-tailed for $p=0.03$ as well as for $p=0.5$ [4] not developing sharp peaks when $L$ is increased. Spin glass behavior occurred in low concentrations $(p=0.03)$ as shown in Figure 4. In Figure 3 the distribution peak increases with the increase of lattice size and it's value scale is $L^{3}$. But in Figures 4-7, the distribution peak goes higher for some $L$ and after that it decreases with increasing value of $L$. This behavior of distributions tells us that spin glass phase occurs in random weak disorder. The aforementioned system is symmetrical within the range $0.5 \leq p \leq 1$ as $0 \leq p \leq 0.5$.

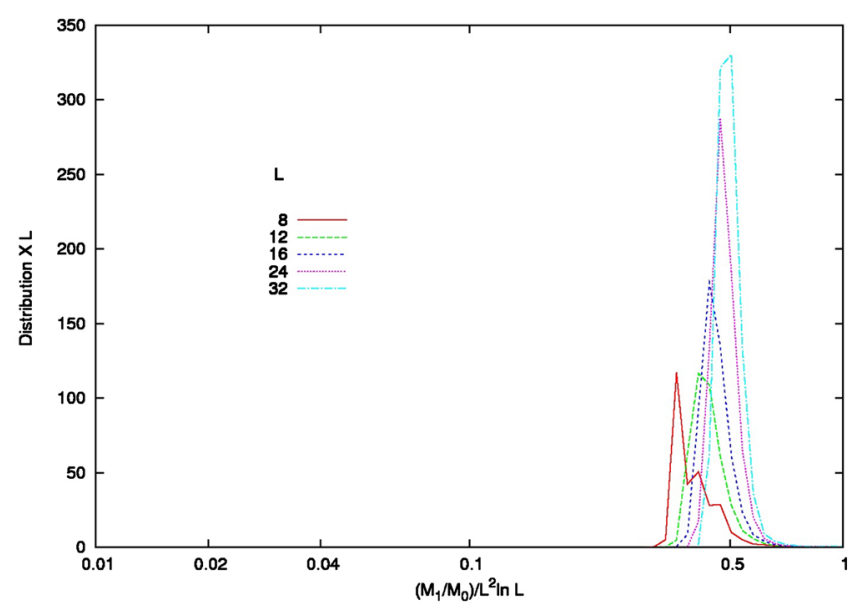

Figure 2. At concentration of defect bonds $p=0.01$, the distribution of $\left(M_{1} / M_{0}\right) L^{2} \ln L$ along $\mathrm{y}$-axis with several lattice sizes and $10^{4}$ disorder realizations.

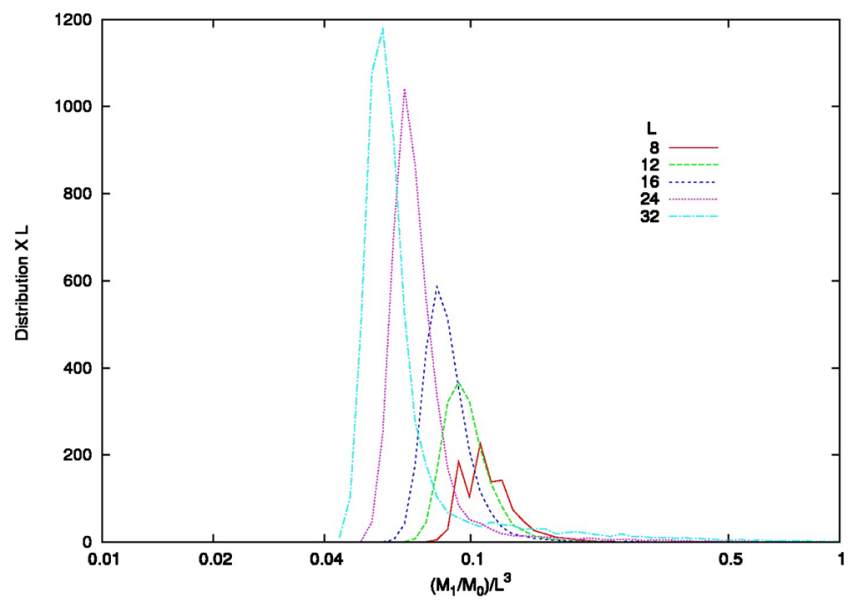

Figure 3. At concentration of defect bonds $p=0.02$, the distribution of $\left(M_{1} / M_{0}\right) L^{3}$ along $\mathrm{y}$-axis with several lattice sizes and $10^{4}$ disorder realizations. 


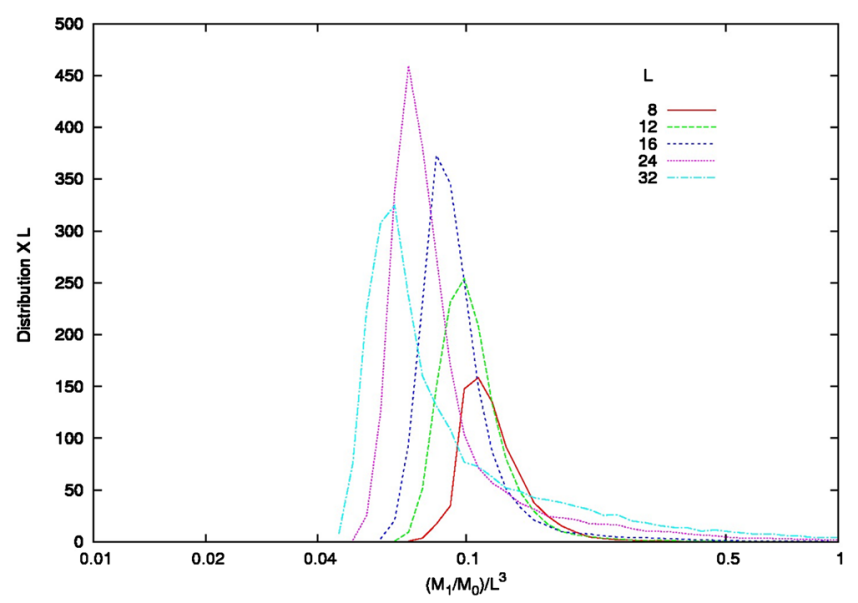

Figure 4. At concentration of defect bonds $p=0.03$, the distribution of $\left(M_{1} / M_{0}\right) L^{3}$ along $\mathrm{y}$-axis with several lattice sizes and $10^{4}$ disorder realizations.

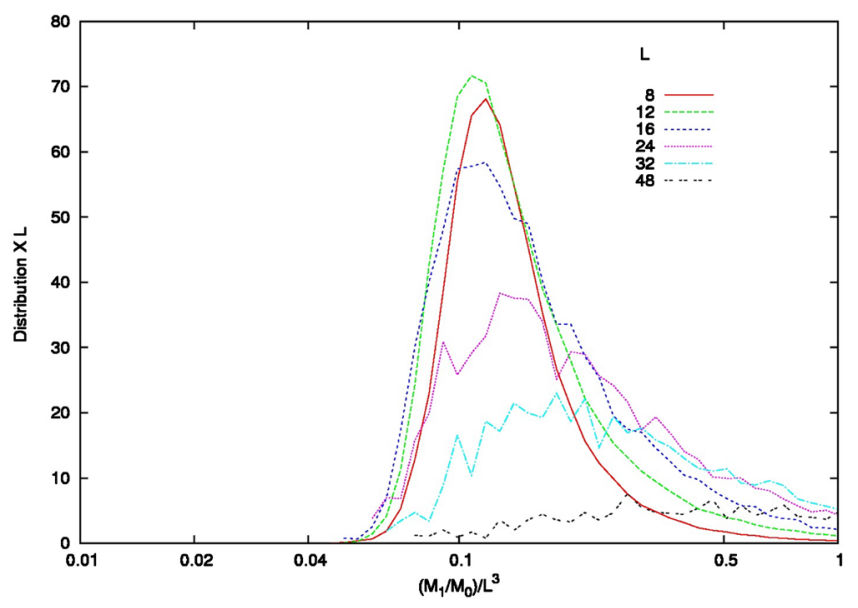

Figure 5. At concentration of defect bonds $p=0.1$, the distribution of $\left(M_{1} / M_{0}\right) L^{3}$ along $\mathrm{y}$-axis with several lattice sizes and $10^{4}$ disorder realizations.

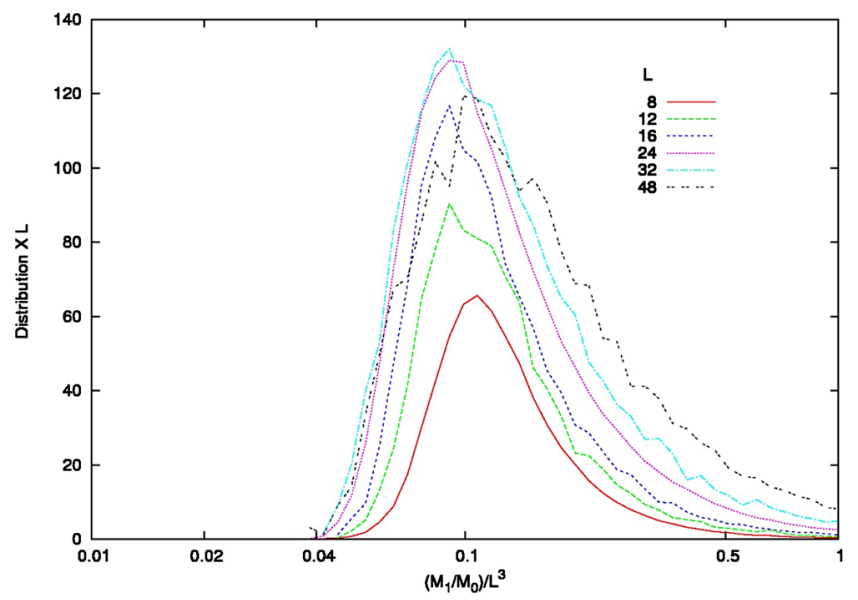

Figure 6. At concentration of defect bonds $p=0.2$, the distribution of $\left(M_{1} / M_{0}\right) L^{3}$ along $y$-axis with several lattice sizes and $10^{5}$ disorder realizations, except for $L=48$ where it was $10^{4}$. 


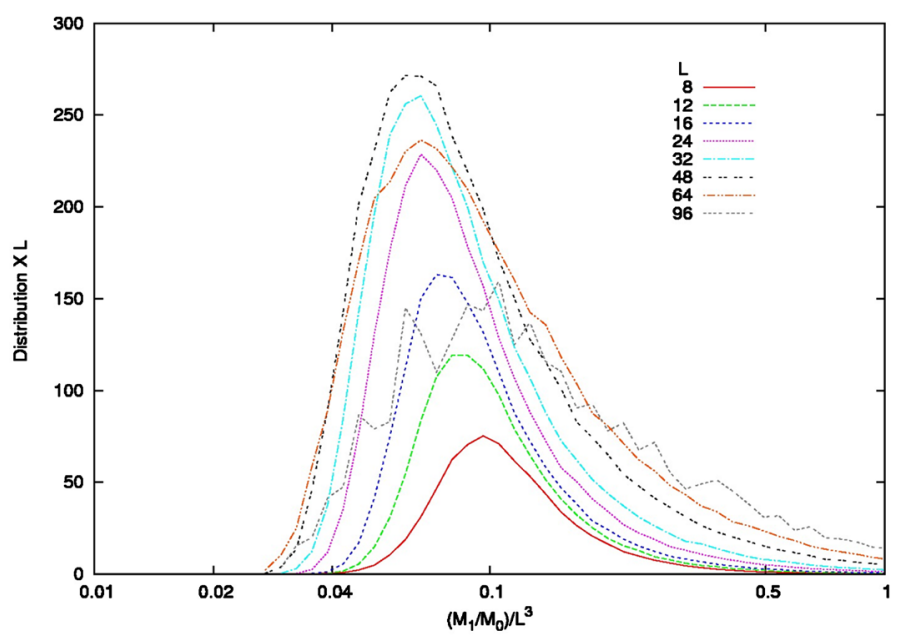

Figure 7. At concentration of defect bonds $p=0.5$, the distribution of $\left(M_{1} / M_{0}\right) L^{3}$ along $y$-axis with several lattice sizes and $10^{5}$ disorder realizations, except for $L=96$ where it was 20,000.

\section{Conclusion}

We have carried out a numerical study of the Villain model, showing that there is an observation that indicates a zero temperature spin glass phase with weak disorder. We have used a computer code that is able to collect numerical data for simulation. The Pfaffian approach is used to collect data for first excitations of ground states, based on perturbation theory. It has been observed clearly that the spin glass phase occurs at concentration of defect bonds $0.03 \leq p \leq 0.5$.

\section{Acknowledgements}

The authors are grateful to the authority of Chittagong University of Engineering and Technology (CUET). The authors also would like to thanks Professor Dr. Julian Poulter for valuable advise during the research work.

\section{References}

[1] Villain, J. (1977) Spin Glass with Non-Random Interactions. Journal of Physics C: Solid State Physics, 10, 1717. http://dx.doi.org/10.1088/0022-3719/10/10/014

[2] Ali, M.Y. and Poulter, J. (2013) Spin Correlation Function of the Fully Frustrated Ising Model and $\pm J$ Ising Spin Glass on the Square Lattice. Chinese Physics B, 22, Article ID: 067502. http://dx.doi.org/10.1088/1674-1056/22/6/067202

[3] Mezard, M., et al. (1987) Spin Glass Theory and Beyond. World Scientific, Singapore.

[4] Atisattapong, W. and Poulter, J. (2008) Energy Gap of the Bimodal Two-Dimensional Ising Spin Glass. New Journal of Physics, 10, Article ID: 093012. http://dx.doi.org/10.1088/1367-2630/10/9/093012

[5] Jinuntuya, N. and Poulter, J. (2012) Elementary Excitations and the Phase Transition in the Bimodal Ising Spin Glass Model. Journal of Statistical Mechanics: Theory and Experiment, 2012, Article ID: P01010. http://dx.doi.org/10.1088/1742-5468/2012/01/P01010

[6] Blackman, J.A. and Poulter, J. (1991) Gauge-Invariant Method for the $\pm J$ Spin-Glass Model. Physical Review B, 44, 4374. http://dx.doi.org/10.1103/PhysRevB.44.4374

[7] Katzgraber, H.G., et al. (2007) Effective Critical Behavior of the Two Dimensional Ising Spin Glass with Bimodal Interaction. Physical Review B, 75, Article ID: 014412.

[8] Kac, M. and Ward, J.C. (1952) A Combinatorial Solution of the Two Dimensional Ising Model. Physical Review, 88, 1332. http://dx.doi.org/10.1103/PhysRev.88.1332

[9] Green, H.S. and Hurst, C.A. (1964) Order-Disorder Phenomena. In-Terscience, London.

[10] Blackman, J.A. and Poulter, J. (1982) Two-Dimensional Frustrated Ising Network as an Eigenvalue Problem. Physical Review B, 26, 4987. http://dx.doi.org/10.1103/PhysRevB.26.4987 
[11] Wang, J.S. and Swendens, R.H. (1988) Monte Carlo Renormalization Group Study of Ising Spin Glasses. Physical Review B, 38, 4840. http://dx.doi.org/10.1103/PhysRevB.37.7745

[12] Fischer, K.S. and Hertz, J.A. (1991) Spin Glasses. Cambridge University Press, London. http://dx.doi.org/10.1017/CBO9780511628771

[13] Stein, D. (1992) Spin Glasses and Biology. World Scientific, Singapore. http://dx.doi.org/10.1142/0446

[14] Sherrington, D. Spin Glasses. Preprint: cond-mat/9806289

[15] Pathria, R.K. (1972) Statistical Mechanics. Pergamon Press, New York. 
Scientific Research Publishing (SCIRP) is one of the largest Open Access journal publishers. It is currently publishing more than 200 open access, online, peer-reviewed journals covering a wide range of academic disciplines. SCIRP serves the worldwide academic communities and contributes to the progress and application of science with its publication.

Other selected journals from SCIRP are listed as below. Submit your manuscript to us via either submit@scirp.org or Online Submission Portal.
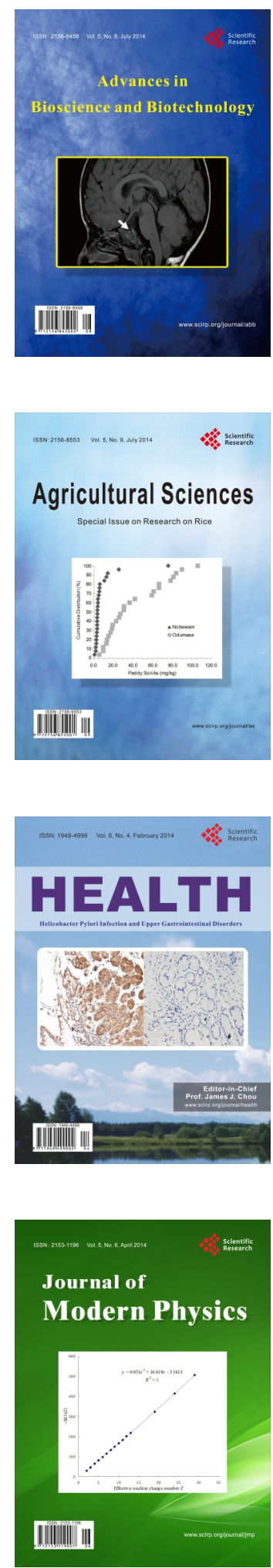
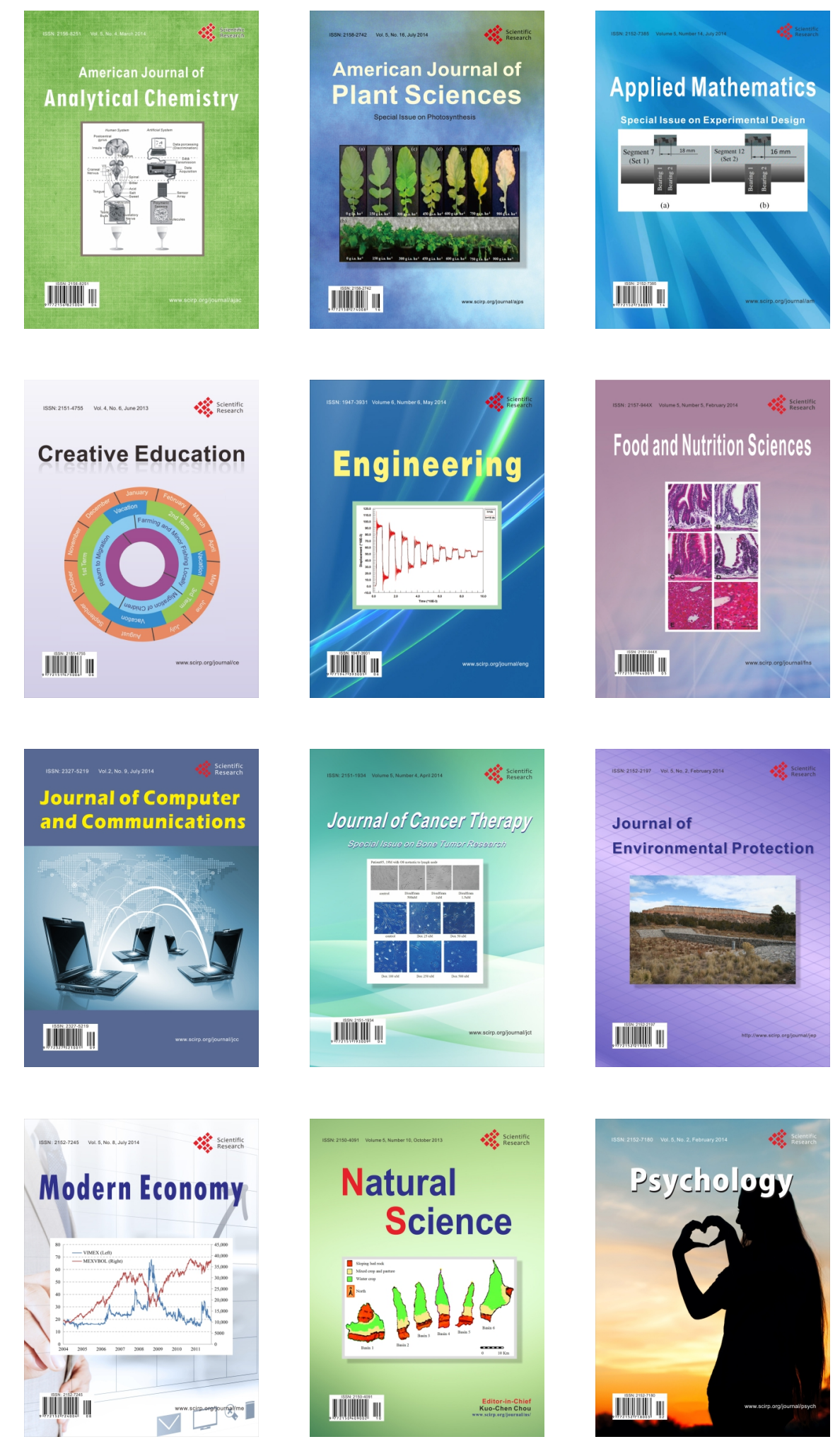